sätzlichen Pauschale bedarf es Darlegungen des Krankenhausträgers, die über die an der Diagnose orientierte Rechnungslegung hinausgehen und eine betriebswirtschaftliche Analyse fordern. Es kann aber erwartet werden, dass nach Abschluss der Analyse, wenn alle Umstände feststehen, aus denen sich Ansprüche für ein abgeschlossenes Wirtschaftsjahr ergeben können, etwaige Forderungen geltend gemacht werden. Das folgt bereits aus dem Gebot der Rücksichtnahme auf die berechtigten Belange der anderen Seite im Rahmen der Dauerbeziehung zwischen Krankenhausträger und Krankenkassen. Diese Verpflichtung schließt es aus, bis zum Ablauf der vierjährigen Verjährung mit der Antragstellung zu warten und den Krankenkassen damit keinen Hinweis auf die Notwendigkeit von Rückstellungen zu geben. Dabei ist unerheblich, aus welchen Gründen die Geltendmachung eines Anspruchs unterblieben ist. Soweit die Beigel. zu 1. hier vorträgt, sie habe angesichts der Haltung der Krankenkassen eine frühere Geltendmachung nicht für erfolgversprechend gehalten, ist dies im Übrigen jedenfalls seit der Einführung der Schlichtungsregelung nicht ohne Weiteres plausibel.

Diese Beurteilung entspricht der Rechtsprechung des Senats, wonach im Rahmen der Vergütung ambulanter vertragsärztlicher Leistungen möglichst Verwerfungen zu vermeiden sind, die dadurch entstehen, dass die aktuelle Gesamtvergütung mit Zahlungen für Leistungen aus lange zurückliegenden Quartalen belastet wird. Grundsätzlich haben sowohl die Vertragsärzte als auch die die Gesamtvergütung entrichtenden Krankenkassen einen Rechtsanspruch darauf, dass die für ein bestimmtes Quartal geleistete Gesamtvergütung möglichst ungeschmälert für die Honorierung der in diesem Quartal erbrachten Leistungen verwendet wird. Das determiniert auch die Ausübung des Ermessens im Rahmen des $₫ 44$ Abs. 2 SGB X bei der Korrektur bestandskräftiger Honorarbescheide (vgl. BSG, SozR 4-1300 \$44 Nr. 6, Rdnrn. 17 ff.).

https://doi.org/10.1007/s00350-017-4828-1

\section{Anmerkung zu BSG Urt. v. 10.5.2017 - B 6 KA 10/16 R (LSG Rheinland-Pfalz)}

\section{Kerrin Schillhorn}

Der 6. Senat hat in seiner Entscheidung v. 10.5.2017 die Gelegenheit genutzt, die Rechtsfigur der Verwirkung über die Krankenhausvergütung auf der Grundlage des KHEntgG hinaus auch dann für anwendbar zu erklären, wenn Krankenhäuser im Rahmen der vertragsärztlichen Vergütung Leistungen abrechnen bzw. Pauschalen vereinbaren können. Durch die Übernahme der Argumentation des 1. Senats des BSG, dass die Beteiligten aufgrund eines dauerhaften Vertragsrahmens ständig professionell zusammenarbeiten, erstreckt der 6 . Senat hier das Rechtsinstrument der Verwirkung auch auf die Vereinbarung von Pauschalen nach $\$ 120$ Abs. 1a SGB V. Auch für derartige Pauschalen, deren rückwirkende Vereinbarkeit der 6. Senat zunächst ausführlich thematisiert, soll die kurze Verwirkungsfrist gelten. Dabei lässt der Senat leider offen, um welchen konkreten Zeitraum es sich handelt. Abgestellt wird lediglich darauf, dass ,erwartet werden [könne], dass er jedenfalls im Folgejahr nach Abschluss der Bilanzierung des vergangenen Jahres und Feststellung des Jahresabschlusses gegenüber den Kos-

Rechtsanwältin Dr. iur. Kerrin Schillhorn, MIL,

Fachanwältin für Verwaltungsrecht und

Fachanwältin für Medizinrecht,

michels.pmks Rechtsanwälte Partnerschaft mbB,

Hohenstaufenring 57, 50674 Köln, Deutschland tenträgern die Forderung nach zusätzlichen Pauschalen gem. \120 Abs. 1a SGB V geltend macht"

Im Ergebnis wird dies dazu führen, dass Kostenträger jegliche Forderungen von Krankenhäusern zum Ende des Folgejahres ablehnen werden, um Pauschalen nach $\$ 120$ Abs. 1a SGB V zu vereinbaren.

Eine solche Erstreckung mag auf den ersten Blick für den Fall des $\$ 120$ Abs. 1a SGB V vertretbar erscheinen. Denn es handelt sich hier um einen Ausgleich der durch die Umstellung der Krankenhausfinanzierung erfolgten Unterdeckung durch den Entfall der Quersubventionierung für die ambulante Behandlung von Kindern und Jugendlichen im Krankenhaus. Nach dem Gesetzeswortlaut soll eine Pauschale deshalb nur dann vereinbart werden können, wenn diese erforderlich ist, um die ambulante Versorgung überhaupt gewährleisten zu können. Tatsächlich sollte ein Krankenhausträger in der Lage sein, noch während des laufenden Jahres, spätestens aber nach $\mathrm{Ab}$ schluss des jeweiligen Jahresabschlusses im Folgejahr entscheiden zu können, ob die Voraussetzungen für die Vereinbarung einer solchen Pauschale in seinem Krankenhaus erfüllt sind. Insoweit scheint die nun durch den 6. Senat getroffene Rechtsprechung und Erweiterung der Verwirkungsanforderungen an Krankenhäuser eine gewisse innere Berechtigung aufzuweisen. Bei näherer Betrachtung muss jedoch feststellt werden, dass es in der hier entschiedenen Konstellation an einem Verwirkungsverhalten, wie z.B. der Übermittlung einer vorbehaltlosen Schlussrechnung, fehlt. Ein solcher Anknüpfungspunkt für die Verwirkung, der in der höchstrichterlichen Rechtsprechung als unerlässlich angesehen wird, vgl. z.B. BSG Urt. v. 5. 7.2016 - B 1 KR 40/15 R -, fehlt in dem hier entschiedenen Sachverhalt. Die Möglichkeit der Vereinbarung von Pauschalen steht den Krankenhäusern aufgrund Gesetzes zu. Weitere „besondere Umstände“, die die Annahme einer Verwirkung begründen könnten, sind nicht ersichtlich. Damit erscheint die Übertragung der Rechtsprechung zur Verwirkung in Bezug auf die Geltendmachung von nachträglichen Korrekturen von Vergütungsansprüchen auf die Vereinbarung nach $₫ 120$ Abs. 1a SGB V aus rechtlicher Sicht eher problematisch.

Mit einer gewissen Sorge muss zudem beobachtet werden, dass das rechtliche Korsett der Krankenhausträger nicht nur durch den Gesetzgeber, sondern auch durch die Rechtsprechung des Bundessozialgerichts zunehmend enger geschnürt wird. So ist insbesondere durch das Rechtsinstrument der Verwirkung der gesetzlich vorgegebene Verjährungsrahmen von 4 Jahren auf höchstens zwei Jahre verkürzt worden.

Es ist nicht recht ersichtlich, aus welchem Grund die Rechtsprechung hier unter Abweichung der gesetzlich normierten Verjährungsfristen eine solche Einengung der Durchsetzung von Ansprüchen durch die Krankenhäuser vornimmt. Dies gilt insbesondere deshalb, da korrespondierende Einschränkungen für die Kostenträger nach wie vor nicht gelten. Insoweit ist der sowohl vom 1. als auch vom 6. Senat vorgenommene Rückgriff auf die Vertrauensgrundlage zwischen Krankenhaus und Krankenkasse aufgrund ihrer dauerhaften Vertragsrahmens der Zusammenarbeit bestehenden Rücksichtnahmegebot hier doch wieder einmal - nur einseitig angewandt worden. Dies gilt auch dann, wenn man, wie das BSG, berücksichtigt, dass jeweils nur die Krankenhäuser über alle für die Abrechnung oder hier Wirtschaftlichkeit der Versorgung maßgeblichen Informationen verfügen. Denn während Krankenkassen zunehmend dazu übergehen, Krankenausabrechnungen bis zur Grenze der Verjährungsfrist rückwirkend zu überprüfen und zu „korrigieren“, ist dieser zeitliche Rahmen den Krankenhäusern für Korrekturen aus ihrer Sicht verwehrt. $\mathrm{Ob}$ dies dem Rücksichtnahmegebot in jeder Hinsicht gerecht wird, erscheint zumindest offen. 